\title{
On Specification Logics for Algebra-Coalgebra Structures: Reconciling Reachability and Observability
}

\author{
Corina Cîrstea $^{\star}$ \\ Oxford University Computing Laboratory \\ Wolfson Building, Parks Road \\ Oxford OX1 3QD, UK \\ Corina.Cirstea@comlab.ox.ac.uk
}

\begin{abstract}
The paper builds on recent results regarding the expressiveness of modal logics for coalgebras in order to introduce a specification framework for coalgebraic structures which offers support for modular specification. An equational specification framework for algebraic structures is obtained in a similar way. The two frameworks are then integrated in order to account for structures comprising both a coalgebraic (observational) component and an algebraic (computational) component. The integration results in logics whose sentences are either coalgebraic (modal) or algebraic (equational) in nature, but whose associated notions of satisfaction take into account both the coalgebraic and the algebraic features of the structures being specified. Each of the logics thus obtained also supports modular specification.
\end{abstract}

\section{Introduction}

In studying structures that involve construction (e.g. data types), one typically uses algebras and their underlying equational logic for specification and reasoning. Such use is supported by the existence of characterisability results for classes of algebras, both in the concrete setting of many-sorted algebras [3] and in a more abstract, categorical setting [21], whereby equationally-specifiable classes of algebras coincide with varieties (that is, classes of algebras closed under subalgebras, homomorphic images and product:1 1 ). In recent years, coalgebras (the categorical duals of algebras) have been used to study structures that involve observation (e.g. systems with state) 1416, and various modal logics have been used to specify and reason about such structures $13 \mid 15$ |10/7]. Moreover, the results in 2] have been dualised in [9, where it was shown that modally-definable classes of coalgebras coincide with covarieties (the duals of varieties).

A framework which integrates algebraic and coalgebraic specification methods in order to specify structures comprising both computational and observational features was described in [5]. The approach taken there was to clearly

\footnotetext{
* Research supported by St. John's College, Oxford.

${ }^{1}$ In an abstract setting, the notions of subalgebra and homomorphic image are defined relatively to a factorisation system for the category of algebras in question.
} 
separate the two categories of features, and to use algebra and respectively coalgebra for specifying them. Such an approach yielded an algebraically-defined notion of reachability under computations, as well as a coalgebraically-defined notion of indistinguishability by observations. Equational, either algebraic or coalgebraic sentences were then used to formalise correctness properties of combined structures, with both algebraic and coalgebraic features playing a rôle in defining the associated notions of satisfaction. Both notions of satisfaction were shown to give rise to institutions 6 .

The equational sentences used in [5], although similar in their expressiveness to the equational formulae of 2 and the modal formulae of [9], have a strong semantic flavour, being indexed by classes of algebras and respectively coalgebras. This makes such sentences difficult to use for actual specification. In addition, the coalgebraic framework described in [5] only considers (coalgebras of) $\omega^{\text {op }}$-continuous, pullback-preserving endofunctors, and thus does not account for endofunctors defined in terms of powersets. Similar $\omega$-cocontinuity restrictions are imposed to the endofunctors used in 5 to specify algebraic structures. A first goal of this paper is to define individual frameworks for the specification of coalgebraic and respectively algebraic structures, which, on the one hand, have a more concrete notion of syntax associated to them, and, on the other hand, are more general than the ones in [5] w.r.t. the endofunctors considered. Then, a second goal of the paper is to integrate the resulting frameworks in order to account for structures having both a coalgebraic and an algebraic component.

In the first part of the paper, coalgebraic and respectively algebraic structures are considered independently of each other. In each case, an institution is shown to arise from suitable choices for the notions of signature, signature morphism, sentence and satisfaction (with the choices for the notion of sentence being driven by the results in 9] and respectively [2]). In the second part of the paper, structures comprising both observational and computational features are considered. As in [5], the choice of models incorporating both coalgebraic and algebraic structure, and of the syntax used to specify these models is based on the approach in [17, and results in a compatibility between the two categories of features, in that computations preserve observational indistinguishability whereas observations preserve reachability. The sentences used for specification are the ones employed by the individual frameworks, while the associated notions of satisfaction exploit the previously-mentioned compatibility in order to abstract away unwanted detail (in the form of unreachable and respectively observationally indistinguishable behaviours). This results in the availability of inductive and respectively coinductive techniques for correctness proofs. Suitably restricting the notions of signature morphism associated to the individual frameworks yields institutions w.r.t. each of the two notions of satisfaction.

\section{Coalgebras and Modal Logic}

This section builds on the approach in [9] in order to obtain notions of cosignature, modal formula and satisfaction of a modal formula by a coalgebra, and 
subsequently derives a specification logic for coalgebras based on these notions. The multi-modal logic described in [7] is used to exemplify the approach.

Definition 1. A cosignature is a pair (C, G), with $\mathrm{C}$ a category and $\mathrm{G}$ an endofunctor on $\mathrm{C}$, subject to the following constraints:

1. C is complete, cocomplete, regula 2 , wellpowered and has enough injectives;

2. G preserves weak pullback 3 ;

3. the functor $\mathrm{U}_{\mathrm{G}}: \operatorname{Coalg}(\mathrm{G}) \rightarrow \mathrm{C}$ which takes $\mathrm{G}$-coalgebras to their carrier has a right adjoint $\mathrm{R}_{\mathrm{G}}$.

G-coalgebras are taken as models for a cosignature $(C, G)$. A consequence of Def.1 and of [8, Prop. 2.1] is that the functor $U_{G}$ is comonadic, and hence Coalg $(\mathrm{G}) \simeq$ Coalg $(\mathrm{D})$ for some comonad $(\mathrm{D}, \epsilon, \delta)$. Specifically, $\mathrm{D}: \mathrm{C} \rightarrow \mathrm{C}$ is given by $\mathrm{U}_{\mathrm{G}} \circ \mathrm{R}_{\mathrm{G}}, \epsilon: \mathrm{D} \Rightarrow$ Id is given by the counit of the adjunction $\mathrm{U}_{\mathrm{G}} \dashv \mathrm{R}_{\mathrm{G}}$, while $\delta: \mathrm{D} \Rightarrow \mathrm{D} \circ \mathrm{D}$ is given by $\mathrm{U}_{\mathrm{G}} \eta_{\mathrm{R}_{\mathrm{G}}}$, with $\eta: \mathrm{Id} \Rightarrow \mathrm{R}_{\mathrm{G}} \circ \mathrm{U}_{\mathrm{G}}$ denoting the unit of $\mathrm{U}_{\mathrm{G}} \dashv \mathrm{R}_{\mathrm{G}}$.

Remark 1. The following hold for a cosignature $(C, G)$ :

1. $U_{G}$ preserves and reflects monomorphisms. This is a consequence of Def.1 (2).

2. The components of $\eta$ are monomorphisms. This follows from $\mathrm{U}_{\mathrm{G}} \eta_{\gamma}$ being a split monomorphism (as $\epsilon_{C} \circ \mathrm{U}_{\mathrm{G}} \eta_{\gamma}=1_{C}$ ) for any G-coalgebra $\langle C, \gamma\rangle$.

3. Coalg $(G)$ has a final object, given by $R_{G} 1$. The final $G$-coalgebra incorporates all abstract G-behaviours. The homomorphisms into it abstract away the non-observable information contained in arbitrary coalgebras.

4. Largest bisimulations on G-coalgebras are constructed as kernel pairs of the C-arrows underlying the unique homomorphisms into the final G-coalgebra. Again, this is consequence of Def.1(2).

Definition 2. Given a cosignature (C, G), a G-coalgebra is observable4 if and only if its unique homomorphism into the final $\mathrm{G}$-coalgebra is a monomorphism.

Example 1. Finitely-branching transition systems are specified using the cosignature $\left(\right.$ Set, $\mathrm{G}_{\mathrm{TS}}$ ), where $\mathrm{G}_{\mathrm{TS}}=\mathcal{P}_{f}(\mathrm{Id})$ (with the functor $\mathcal{P}_{f}:$ Set $\rightarrow$ Set taking a set to the set of its finite subsets). Finitely-branching, $A$-labelled transition systems are specified using the cosignature (Set, $\left.\mathrm{G}_{\mathrm{LTS}}\right)$, where $\mathrm{G}_{\mathrm{LTS}}=\mathcal{P}_{f}(A \times \mathrm{Id})$.

The following is a direct consequence of [8, Lemma 3.9].

Proposition 1. For a cosignature $(\mathrm{C}, \mathrm{G})$, the category Coalg $(\mathrm{G})$ is regular, and the functor $\mathrm{U}_{\mathrm{G}}$ preserves regular-epi-mono factorisations.

${ }^{2}$ Hence, $\mathrm{C}$ has regular-epi-mono factorisations. The existence of strong-epi-mono factorisations (which is a consequence of the completeness and wellpoweredness of $\mathrm{C}$ ) is actually sufficient for the approach in this section. Regularity is only required here for consistency with the next two sections.

${ }^{3}$ Weak pullbacks are defined similarly to standard pullbacks, except that the mediating arrow is not required to be unique.

${ }^{4}$ Observable coalgebras are called simple in [16. 
The existence of a factorisation system for Coalg $(G)$ yields notions of $G$ subcoalgebra (given by a monomorphism in Coalg $(\mathrm{G})$ ), G-homomorphic image (given by a regular epimorphism in Coalg $(\mathrm{G})$ ), and $\mathrm{G}$-covariety (given by a class of G-coalgebras which is closed under subcoalgebras, homomorphic images and coproduct 5 ). A characterisability result for covarieties in terms of modal formulae is given in [9]. There, covarieties are defined in terms of factorisation systems $(\mathcal{E}, \mathcal{M})$ for categories of coalgebras, while modal formulae are defined as subcoalgebras with $\mathcal{M}$-injective codomains. However, as noted in [9. Thm. 2.5.4], in order to characterise covarieties it suffices to consider modal formulae given by subcoalgebras whose codomains belong to a subclass of $\mathcal{M}$-injective objects, namely a subclass which provides enough $\mathcal{M}$-injectives for the category of coalgebras in question. The notion of covariety considered here is obtained by instantiating the one in $[9$ with the factorisation system $(\operatorname{RegEpi}(\operatorname{Coalg}(G))$, Mono $(\operatorname{Coalg}(G)))$. The following observations can be made relatively to this factorisation system:

1. If a C-object $Z$ is injective (and hence $U_{G}(\operatorname{Mono}(\operatorname{Coalg}(G))$ )-injective), then the $\mathrm{G}$-coalgebra $\mathrm{R}_{\mathrm{G}} Z$ is injective (by [9, Prop. 2.2.10]).

2. If $C$ has enough injectives, then $\operatorname{Coalg}(G)$ has enough injectives. Moreover, the cofree $\mathrm{G}$-coalgebras over injective $\mathrm{C}$-objects still provide enough injectives for Coalg(G). For, if $\langle C, \gamma\rangle$ is a G-coalgebra and $f: C \rightarrow Z$ is a Mono(C)arrow with an injective codomain, then $f^{b}=\mathrm{R}_{\mathrm{G}} f \circ \eta_{\gamma}:\langle C, \gamma\rangle \rightarrow \mathrm{R}_{G} Z$ is a Mono(Coalg $(G)$ )-arrow with an injective codomain. (Rem.11(2) and the preservation of monomorphisms by $\mathrm{R}_{\mathrm{G}}$ are used here.)

These observations together with the previous remarks justify the following.

Definition 3. A modal formula over a cosignature (C, G) is a $\mathrm{G}$-subcoalgebra $\iota:\langle D, \delta\rangle \rightarrow \mathrm{R}_{\mathrm{G}} Z$ with $Z$ an injective $\mathrm{C}$-object. A G-coalgebra $\langle C, \gamma\rangle$ satisfies a modal formula of this form (written $\langle C, \gamma\rangle=\iota$ ) if and only if, for any $\mathrm{C}$-arrow $f: C \rightarrow Z$, the $\mathrm{G}$-coalgebra homomorphism $f^{b}:\langle C, \gamma\rangle \rightarrow \mathrm{R}_{\mathrm{G}} Z$ factors through $\iota$.

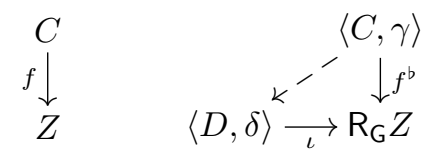

Modal formulae $\iota:\langle D, \delta\rangle \rightarrow \mathrm{R}_{\mathrm{G}} Z$ with $Z$ final in $\mathrm{C}$ (and hence $\mathrm{R}_{\mathrm{G}} Z$ final in Coalg $(G)$ ) specify properties up to observational equivalence. Existing specification frameworks for coalgebras [13 15 10 7] only employ such modal formulae.

The satisfaction of modal formulae by coalgebras is preserved along homomorphic images and reflected along subcoalgebras. These are consequences of [9. Coroll. 2.4.6] and respectively [9, Prop. 2.4.8], but also follow directly from Def.3 together with Prop.1] Also, a consequence of [9, Coroll. 2.5.5] and of the dual of [1. Coroll. 20.29] is that, for a cosignature (C, G), modally definable 6 classes of G-coalgebras coincide with covarieties.

\footnotetext{
${ }^{5}$ Note that the cocompleteness of $\mathrm{C}$ results in the cocompleteness of Coalg $(\mathrm{G})$, with colimits in Coalg $(\mathrm{G})$ being created by $\mathrm{U}_{\mathrm{G}}$.

${ }^{6}$ In the sense of Def. 3
} 
We now show that monomorphisms into the carriers of coalgebras induce largest subcoalgebras factoring through such monomorphisms.

Proposition 2. Let $(\mathrm{C}, \mathrm{G})$ denote a cosignature, let $\langle C, \gamma\rangle$ denote a $\mathrm{G}$-coalgebra, and let $\iota: X \rightarrow C$ denote a C-monomorphism. Then, the full subcategory of Coalg $(\mathrm{G}) /\langle C, \gamma\rangle$ whose objects $\langle\langle D, \delta\rangle, d\rangle$ are such that $\mathrm{U}_{\mathrm{G}}$ d factors through $\iota$ has a final object $\langle\langle E, \eta\rangle, m\rangle$. Moreover, $m$ defines a G-subcoalgebra of $\langle C, \gamma\rangle$.

Proof (sketch). Since $\mathrm{C}$ is wellpowered, there is only a set $\mathcal{D}$ of $\mathrm{G}$-coalgebra homomorphisms $d:\langle D, \delta\rangle \rightarrow\langle C, \gamma\rangle$ whose image under $\mathrm{U}_{\mathrm{G}}$ is a monomorphism which factors through $\iota$. If $c: \coprod_{d \in \mathcal{D}} \operatorname{dom}(d) \rightarrow\langle C, \gamma\rangle$ is the G-coalgebra homomorphism arising from the universality of $\coprod_{d \in \mathcal{D}} \operatorname{dom}(d)$, and if $c=m \circ e$ is a regular-epimono factorisation for $c$, then one can show that $m$ defines a final object in the full subcategory of Coalg $(\mathrm{G}) /\langle C, \gamma\rangle$ whose objects $\langle\langle D, \delta\rangle, d\rangle$ are such that $\mathrm{U}_{\mathrm{G}} d$ factors through $\iota$. (The preservation of coproducts and of regular-epi-mono factorisations by $\mathrm{U}_{\mathrm{G}}$ and the unique $(\operatorname{RegEpi}(\mathrm{C})$, Mono $(\mathrm{C})$ )-diagonalisation property of $\mathrm{C}$ are used.)

Similarly, modal formulae induce largest subcoalgebras of given coalgebras.

Proposition 3. Let $(\mathrm{C}, \mathrm{G})$ denote a cosignature, let $\langle C, \gamma\rangle$ denote a $\mathrm{G}$-coalgebra, and let $\mathcal{F}$ denote a set of modal formulae over $(\mathrm{C}, \mathrm{G})$. Then, the full subcategory of Coalg $(\mathrm{G}) /\langle C, \gamma\rangle$ whose objects satisfy the modal formulae in $\mathcal{F}$ has a final object, which at the same time defines a G-subcoalgebra of $\langle C, \gamma\rangle$.

Proof. Similar to the proof of Prop.2. The set $\mathcal{D}$ of G-coalgebra homomorphisms $d:\langle D, \delta\rangle \rightarrow\langle C, \gamma\rangle$ which are such that $\mathrm{U}_{\mathrm{G}} d$ is a $\mathrm{C}$-monomorphism and such that $f^{b}:\langle D, \delta\rangle \rightarrow \mathrm{R}_{\mathrm{G}} Z$ factors through $\iota$ for any $\iota \in \mathcal{F}$ with codomain $\mathrm{R}_{\mathrm{G}} Z$ and any $f: D \rightarrow Z$ is considered this time.

7] (see also [15) develops a modal logic for coalgebras of (finite) Kripke polynomial endofunctors, that is, endofunctors $G$ on Set constructed from constant and identity endofunctors using products, coproducts, exponentials with constant exponent and (finite) powersets. Here we use finite Kripke polynomial endofunctors (which satisfy the conditions in Def.1, and therefore give rise to cosignatures) to exemplify our approach. The modal formulae associated to such endofunctors, built from basic formulae of form $a$ with $a \in A$ (where the set $A$ appears covariantly in the definition of $G$ ) using propositional connectives and modal operators (whose form depends on $G$ ), induce predicates on the carriers of final G-coalgebras, and therefore, by Prop.2, are an instance of the notion of modal formula given by Def. 3 Moreover, the fact that bisimulation coincides with logical equivalence (see 7, Coroll. 5.9], or [15] Prop. 4.8]) results in the notion of satisfaction used in [7] agreeing with the one given by Def. 3

Example 2. Consider the cosignature (Set, $\left.\mathrm{G}_{\mathrm{FTS}}\right)$, with $\mathrm{G}_{\mathrm{FTS}}=\mathcal{P}_{f}(\mathrm{Id}) \times \mathbb{N}$. Under the approach in [7, the modal language associated to $\mathrm{G}_{\mathrm{FTS}}$ is given by:

$$
\varphi::=\perp|\varphi \rightarrow \psi|[\operatorname{succ}] \varphi\left|[\operatorname{depth}] \varphi_{\mathbb{N}} \quad \varphi_{\mathbb{N}}::=\perp\right| \varphi_{\mathbb{N}} \rightarrow \psi_{\mathbb{N}} \mid n, n \in \mathbb{N}
$$


while its associated notion of satisfaction is given by:

$$
\begin{aligned}
C, n^{\prime}=n & \Leftrightarrow n^{\prime}=n \\
C, c \models[\operatorname{succ}] \varphi & \Leftrightarrow(\forall s)(s \in \operatorname{succ}(c) \Rightarrow C, s \models \varphi) \\
C, c=[\operatorname{depth}] \varphi_{\mathbb{N}} & \Leftrightarrow(\forall n)\left(\operatorname{depth}(c)=n \Rightarrow C, n \models \varphi_{\mathbb{N}}\right)
\end{aligned}
$$

for any $\mathrm{G}_{\mathrm{FTS}}$-coalgebra $C=\left\langle C,\left\langle\right.\right.$ succ $: C \rightarrow \mathcal{P}_{f}(C)$, depth $\left.\left.: C \rightarrow \mathbb{N}\right\rangle\right\rangle, n, n^{\prime} \in \mathbb{N}$ and $c \in C$. One can also define modal operators $\langle$ succ $\rangle$ and $\langle$ depth $\rangle$, namely by $\langle\operatorname{succ}\rangle \varphi::=\neg[$ succ $] \neg \varphi$ and $\langle\operatorname{depth}\rangle \varphi_{\mathbb{N}}::=\neg[\operatorname{depth}] \neg \varphi_{\mathbb{N}}$. Thus:

$$
\begin{aligned}
& C, c \models\langle\operatorname{succ}\rangle \varphi \Leftrightarrow(\exists s)(s \in \operatorname{succ}(c) \text { and } C, s \models \varphi) \\
& C, c \models\langle\operatorname{depth}\rangle \varphi_{\mathbb{N}} \Leftrightarrow(\exists n)\left(\operatorname{depth}(c)=n \text { and } C, n \models \varphi_{\mathbb{N}}\right)
\end{aligned}
$$

(Note therefore that $\langle\operatorname{depth}\rangle \varphi_{\mathbb{N}}$ and $[\operatorname{depth}] \varphi_{\mathbb{N}}$ are semantically equivalent for any $\varphi$.) Finitely branching transition systems of finite depth can now be specified using the following modal formulae:

$$
\begin{gathered}
{[\text { depth }] 0 \leftrightarrow[\text { succ }] \perp} \\
{[\operatorname{depth}](n+1) \leftrightarrow\langle\text { succ }\rangle[\operatorname{depth}] n \wedge[\text { succ }][\operatorname{depth}](0 \vee \ldots \vee n), n \in \mathbb{N}}
\end{gathered}
$$

formalising the statement that a rooted transition system has depth 0 precisely when its root has no successors, and has depth $n+1$ precisely when its root has a successor of depth $n$, and the depth of any of its successors does not exceed $n$. Alternatively, natural numbers can be regarded as colours used to decorate the states of an unlabelled transition system. The decorations of interest are those where the colour decreases by 1 in successor states, and where (only) states with no successor have colour 0 . Such an approach, equivalent to the previous one, corresponds to specifying a subcoalgebra of the cofree $\mathcal{P}_{f}($ Id)-coalgebra over $\mathbb{N}$.

We now introduce a notion of cosignature morphism, capturing translations between different types of observational structures.

Definition 4. A cosignature morphism between cosignatures $(\mathrm{C}, \mathrm{G})$ and $\left(\mathrm{C}^{\prime}, \mathrm{G}^{\prime}\right)$ consists of a pair $(\mathrm{U}, \tau)$ with $\mathrm{U}: \mathrm{C}^{\prime} \rightarrow \mathrm{C}$ a functor with right adjoint $\mathrm{R}$, and with $\tau: \mathrm{U} \circ \mathrm{G}^{\prime} \Rightarrow \mathrm{G} \circ \mathrm{U}$ a natural transformation.

Cosignature morphisms $(\mathrm{U}, \tau):(\mathrm{C}, \mathrm{G}) \rightarrow\left(\mathrm{C}^{\prime}, \mathrm{G}^{\prime}\right)$ induce reduct functors $\mathrm{U}_{\tau}$ : Coalg $\left(\mathrm{G}^{\prime}\right) \rightarrow$ Coalg $(\mathrm{G})$, with $\mathrm{U}_{\tau}$ taking $\left\langle C^{\prime}, \gamma^{\prime}\right\rangle \in\left|\operatorname{Coalg}\left(\mathrm{G}^{\prime}\right)\right|$ to $\left\langle\mathrm{U} C^{\prime}, \tau_{C^{\prime}} \circ \mathrm{U} \gamma^{\prime}\right\rangle \in$ $\mid$ Coalg $(\mathrm{G}) \mid$. Moreover, the existence of cofree coalgebras w.r.t. the functors $U_{\mathrm{G}}$ and $\mathrm{U}_{\mathrm{G}^{\prime}}$, together with the existence of largest subcoalgebras induced by monomorphisms into the carriers of coalgebras (see Prop.2) can be used to show that the reduct functors induced by cosignature morphisms have right adjoints.

Proposition 4. Let $(\mathrm{U}, \tau):(\mathrm{C}, \mathrm{G}) \rightarrow\left(\mathrm{C}^{\prime}, \mathrm{G}^{\prime}\right)$ denote a cosignature morphism. Then, the reduct functor $\mathrm{U}_{\tau}: \operatorname{Coalg}\left(\mathrm{G}^{\prime}\right) \rightarrow \operatorname{Coalg}(\mathrm{G})$ has a right adjoint.

Proof (sketch). A cofree $\mathrm{G}^{\prime}$-coalgebra over a $\mathrm{G}$-coalgebra $\langle C, \gamma\rangle$ w.r.t. $\mathrm{U}_{\tau}$ is obtained as the largest $\mathrm{G}^{\prime}$-subcoalgebra of the cofree $\mathrm{G}^{\prime}$-coalgebra over RC w.r.t. $\mathrm{U}_{\mathrm{G}^{\prime}}$ whose image under $\mathrm{U}_{\tau}$ has a homomorphism into $\langle C, \gamma\rangle$. 
The right adjoint to $\mathrm{U}_{\tau}$ is denoted $\mathrm{R}_{\tau}$. The uniqueness up to isomorphism of right adjoints yields a natural isomorphism $i: \mathrm{R}_{\tau} \circ \mathrm{R}_{\mathrm{G}} \Rightarrow \mathrm{R}_{\mathrm{G}^{\prime}} \circ \mathrm{R}$.

The existence of right adjoints to the reduct functors induced by cosignature morphisms yields translations of modal formulae over the sources of cosignature morphisms to modal formulae over their targets. Specifically, a modal formula $\iota:\langle D, \delta\rangle \rightarrow \mathrm{R}_{\mathrm{G}} Z$ translates along a cosignature morphism $(\mathrm{U}, \tau):(\mathrm{C}, \mathrm{G}) \rightarrow$ $\left(\mathrm{C}^{\prime}, \mathrm{G}^{\prime}\right)$ to the modal formula $\tau(\iota)=i_{Z} \circ \mathrm{R}_{\tau} \iota: \mathrm{R}_{\tau}\langle D, \delta\rangle \rightarrow \mathrm{R}_{\mathrm{G}^{\prime}} \mathrm{R} Z$. (The fact that $\mathrm{R} Z$ is injective whenever $Z$ is injective follows from the preservation of monomorphisms by $\mathrm{U}_{\mathrm{G}}$. Also, the fact that $\mathrm{U}_{\mathrm{G}^{\prime}} \mathrm{R}_{\tau} \iota$, and hence $i_{Z} \circ \mathrm{U}_{\mathrm{G}^{\prime}} \mathrm{R}_{\tau} \iota$, belongs to Mono $\left(C^{\prime}\right)$ follows from $U_{G} \iota$ being a monomorphism together with the reflection of monomorphisms by $U_{G}$ and their preservation by each of $R_{\tau}$ and $U_{G^{\prime}}$.)

The translation of modal formulae along cosignature morphisms is such that the satisfaction condition of institutions (formalising the statement that truth is invariant under changes of notation [6]) holds for the resulting logic.

Theorem 1. Let $(\mathrm{U}, \tau):(\mathrm{C}, \mathrm{G}) \rightarrow\left(\mathrm{C}^{\prime}, \mathrm{G}^{\prime}\right)$ denote a cosignature morphism, let $\left\langle C^{\prime}, \gamma^{\prime}\right\rangle$ denote a $\mathrm{G}^{\prime}$-coalgebra, and let $\iota:\langle D, \delta\rangle \rightarrow \mathrm{R}_{\mathrm{G}} Z$ denote a modal formula $\operatorname{over}(\mathrm{C}, \mathrm{G})$. Then, $\mathrm{U}_{\tau}\left\langle C^{\prime}, \gamma^{\prime}\right\rangle \models \iota$ iff $\left\langle C^{\prime}, \gamma^{\prime}\right\rangle \models \tau(\iota)$.

Proof (sketch).

$$
\left.\mathrm{U}_{\tau}\left\langle C^{\prime}, \gamma^{\prime}\right\rangle=\iota \Leftrightarrow \text { (defn. of }=\right)
$$

for all $f: \mathrm{U} C^{\prime} \rightarrow Z, f^{b}$ factors through $\iota \Leftrightarrow\left(\mathrm{U} \dashv \mathrm{R}, \mathrm{U}_{\tau} \dashv \mathrm{R}_{\tau}, i\right.$-iso $)$

for all $f^{\prime}: C^{\prime} \rightarrow \mathrm{R} Z, f^{\prime \prime}$ factors through $i_{Z} \circ \mathrm{R}_{\tau} \iota \Leftrightarrow($ defn. of $\models)$

$$
\left\langle C^{\prime}, \gamma^{\prime}\right\rangle \models \tau(\iota)
$$

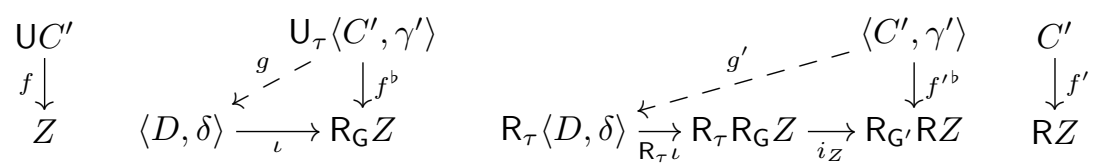

The second equivalence exploits the existence of isomorphisms $\mathrm{C}\left(\mathrm{U} C^{\prime}, Z\right) \simeq$ $\mathrm{C}^{\prime}\left(C^{\prime}, \mathrm{R} Z\right)$ and Coalg $(\mathrm{G})\left(\mathrm{U}_{\tau}\left\langle C^{\prime}, \gamma^{\prime}\right\rangle,\langle D, \delta\rangle\right) \simeq \operatorname{Coalg}\left(\mathrm{G}^{\prime}\right)\left(\left\langle C^{\prime}, \gamma^{\prime}\right\rangle, \mathrm{R}_{\tau}\langle D, \delta\rangle\right)$, and the relationship between the counits of the adjunctions $\mathrm{U} \dashv \mathrm{R}, \mathrm{U}_{\tau} \dashv \mathrm{R}_{\tau}, \mathrm{U}_{\mathrm{G}} \dashv \mathrm{R}_{\mathrm{G}}$ and $U_{\mathrm{G}^{\prime}} \dashv \mathrm{R}_{\mathrm{G}^{\prime}}$, determined by the existence of the natural isomorphism $i$.

Example 3. Consider the cosignature (Set, $\left.\mathrm{G}_{\mathrm{LFTS}}\right)$, with $\mathrm{G}_{\mathrm{LFTS}}=\mathcal{P}_{f}(A \times \mathrm{Id}) \times \mathbb{N}$. Then, $($ Id, $\tau)$ with $\tau=\mathcal{P}_{f}\left(\pi_{2}\right) \times 1_{\mathbb{N}}: \mathcal{P}_{f}(A \times$ Id $) \times \mathbb{N} \Rightarrow \mathcal{P}_{f}($ Id $) \times \mathbb{N}$ defines a cosignature morphism from (Set, $\mathrm{G}_{\mathrm{FTS}}$ ) to (Set, $\left.\mathrm{G}_{\mathrm{LFTS}}\right)$. The induced reduct functor takes a $\mathrm{G}_{\mathrm{LFTS}}$-coalgebra $\langle C,\langle$ next, depth $\rangle\rangle$ to the $\mathrm{G}_{\mathrm{FTS}}$-coalgebra $\left\langle C,\left\langle\mathcal{P}_{f}\left(\pi_{2}\right) \circ\right.\right.$ next, depth $\rangle\rangle$. The modal language associated to $\mathrm{G}_{\mathrm{LFTS}}$ is given by:

$\varphi::=\perp|\varphi \rightarrow \psi|[$ label $] \varphi_{A} \mid[$ succ $] \varphi \mid[$ depth $] \varphi_{\mathbb{N}} \varphi_{A}::=\perp\left|\varphi_{A} \rightarrow \psi_{A}\right| a, a \in A$

while its associated notion of satisfaction is given by:

$$
\begin{aligned}
C, c=[\text { label }] \varphi_{A} & \Leftrightarrow(\forall a)\left(a \in \mathcal{P}_{f}\left(\pi_{1}\right)(\operatorname{next}(c)) \Rightarrow C, a \models \varphi_{A}\right) \\
C, c=[\text { succ }] \varphi & \Leftrightarrow(\forall s)\left(s \in \mathcal{P}_{f}\left(\pi_{2}\right)(\operatorname{next}(c)) \Rightarrow C, s \models \varphi\right) \\
C, c \models[\operatorname{depth}] \varphi_{\mathbb{N}} & \Leftrightarrow(\forall n)\left(\operatorname{depth}(c)=n \Rightarrow C, n \models \varphi_{\mathbb{N}}\right)
\end{aligned}
$$


for any $\mathrm{G}_{\mathrm{LFTS}}$-coalgebra $C=\left\langle C\right.$, next $: C \rightarrow \mathcal{P}_{f}(A \times C)$, depth $\left.\left.: C \rightarrow \mathbb{N}\right\rangle\right\rangle$ and $c \in C$. The translation along (Id, $\tau$ ) of modal formulae over $\mathrm{G}_{\mathrm{FTS}}$ to modal formulae over $\mathrm{G}_{\mathrm{LFTS}}$ leaves $\mathrm{G}_{\mathrm{FTS}}$-formulae (including the ones in Ex.2) unchanged.

Remark 2. Cosignature morphisms $(\mathrm{U}, \tau):(\mathrm{C}, \mathrm{G}) \rightarrow\left(\mathrm{C}^{\prime}, \mathrm{G}^{\prime}\right)$ induce comonad morphisms $7(\mathrm{U}, \rho):(\mathrm{D}, \epsilon, \delta) \Rightarrow\left(\mathrm{D}^{\prime}, \epsilon^{\prime}, \delta^{\prime}\right)$. Specifically, if $\bar{\delta}: \mathrm{D} \Rightarrow \mathrm{G} \circ \mathrm{D}$ and $\overline{\delta^{\prime}}: \mathrm{D}^{\prime} \Rightarrow \mathrm{G}^{\prime} \circ \mathrm{D}^{\prime}$ denote the $\mathrm{G}$ - and respectively $\mathrm{G}^{\prime}$-coalgebra structures on $\mathrm{D}$ and respectively $\mathrm{D}^{\prime}$, then the natural transformation $\rho: \mathrm{U} \circ \mathrm{D}^{\prime} \Rightarrow \mathrm{D} \circ \mathrm{U}$ arises from the cofreeness of $\left\langle\mathrm{D} \circ \mathrm{U}, \bar{\delta}_{\mathrm{U}}\right\rangle$ w.r.t. $\mathrm{U}_{\mathrm{G}}$ : for $C^{\prime} \in\left|\mathrm{C}^{\prime}\right|, \rho_{C^{\prime}}$ is the C-arrow underlying the G-coalgebra homomorphism $\left(\mathrm{U} \epsilon_{C^{\prime}}^{\prime}\right)^{b}:\left\langle\mathrm{UD}^{\prime} C^{\prime}, \tau_{\mathrm{D}^{\prime} C^{\prime}}\right.$ 。 $\left.\mathrm{U} \overline{\delta^{\prime}} C^{\prime}\right\rangle \rightarrow\left\langle\mathrm{DU} C^{\prime}, \bar{\delta}_{\mathrm{U} C^{\prime}}\right\rangle$ (where $\left.\mathrm{U} \epsilon_{C^{\prime}}^{\prime}: \mathrm{UD}^{\prime} C^{\prime} \rightarrow \mathrm{U} C^{\prime}\right)$. The induced comonad morphism provides some information about the relationship between the notions of observability associated to $G$ and $G^{\prime}$. For, if $1^{\prime}$ denotes a final $C^{\prime}$-object, then the C-arrow $\rho_{1^{\prime}}: \mathrm{UD}^{\prime} 1^{\prime} \rightarrow \mathrm{DU} 1^{\prime}$ defines the unique homomorphism from the $\mathrm{U}_{\tau}$-reduct of the final $\mathrm{G}^{\prime}$-coalgebra to the final G-coalgebra. Hence, the fact that $\rho_{1^{\prime}}$ belongs to Mono $(\mathrm{C})$ reflects the fact that the target cosignature does not refine the notion of observability induced by the source cosignature.

Definition 5. A cosignature morphism $(\mathrm{U}, \tau)$ is horizontal if and only if $\rho_{1^{\prime}} \in$ Mono $(\mathrm{C})$, with $(\mathrm{U}, \rho)$ denoting the induced comonad morphism.

\section{Algebras and Equational Logic}

A framework for the specification of algebraic structures can be obtained using an approach similar to that of the previous section.

Definition 6. A signature is a pair (C, F), with $\mathrm{C}$ a category and $\mathrm{F}$ an endofunctor on $\mathrm{C}$, subject to the following constraints:

1. $\mathrm{C}$ is complete, cocomplete, regular, RegEpi(C)-cowellpowered and has enough $\operatorname{Reg} \operatorname{Epi}(\mathrm{C})$-projectives;

2. all regular epimorphisms in $\mathrm{C}$ are split;

3. the functor taking $\mathrm{F}$-algebras to their carrier has a left adjoint.

It then follows by [1, Thm. 20.178 and respectively by [4, Thm. 4.3.5] that, for a signature $(\mathrm{C}, \mathrm{F})$, the functor taking F-algebras to their carrier is monadic and preserves regular epimorphism, 9 . Hence, by [1, Thm. 20.32], this functor is RegEpi $(\mathrm{C})$-monadic. Thus, $\operatorname{Alg}(\mathrm{F}) \simeq \operatorname{Alg}(\mathrm{T})$, with the monad $(\mathrm{T}, \eta, \mu)$ being defined similarly to the comonad $(\mathrm{D}, \epsilon, \delta)$ induced by a cosignature.

$\mathrm{T}$-algebras are taken as models for a signature (C, F 10 . The functor taking $\mathrm{T}$-algebras to their carrier is denoted $\mathrm{U}_{\mathrm{T}}$, while its left adjoint (whose existence is guaranteed by Def.6(3) ) is denoted $L_{T}$.

7 The notion of (co)monad morphism considered here generalises the standard one, as defined e.g. in [1, Def. 20.55], being given by a pair $(\mathrm{U}, \rho)$ with $\mathrm{U}: \mathrm{C}^{\prime} \rightarrow \mathrm{C}$ and $\rho: \mathrm{U} \circ \mathrm{D}^{\prime} \Rightarrow \mathrm{D} \circ \mathrm{U}$, rather than by a natural transformation $\rho: \mathrm{D}^{\prime} \Rightarrow \mathrm{D}$.

${ }^{8}$ See also 9 Thm.1.1.8] for a proof in the dual case.

${ }^{9}$ Note that [4 Thm. 4.3.5] requires Def.6(2) to hold, and the category $C$ to be regular.

10 The choice of working with algebras of the induced monads rather than with algebras of endofunctors is driven by the approach in Section 4 . 
Remark 3. The following hold for a signature (C, F):

1. $\operatorname{Alg}(T)$ is cocomplete. This follows by [4, Thm. 4.3.5]. In particular, Alg(T) has an initial object, which incorporates all ground F-computations. The homomorphisms from it interpret ground computations in arbitrary algebras.

2. $\mathrm{U}_{\mathrm{T}}$ preserves and reflects regular epimorphisms. Again, this follows by 4 Thm. 4.3.5].

3. The components of the counit $\epsilon$ of the adjunction $L_{T} \dashv U_{T}$ are regular epimorphisms (as the components of $U_{T} \epsilon$ are split, and hence regular epimorphisms).

Definition 7. Given a signature (C, F), a T-algebra is reachable if and only if the unique homomorphism from the initial $\mathrm{T}$-algebra into it is a regular epimorphism.

Example 4. Non-deterministic, sequential processes over an alphabet $A$ are specified using the signature (Set, $\mathrm{F}_{\mathrm{NSP}}$ ), with $\mathrm{F}_{\mathrm{NSP}}=1+A \times X+X \times X+X \times X$. An $\mathrm{F}_{\mathrm{NSP}}$-algebra is given by a Set-arrow $\alpha: \mathrm{F}_{\mathrm{NSP}} C \rightarrow C$, or equivalently, by four Set-arrows nil : $1 \rightarrow C, \ldots$. $: A \times C \rightarrow C,_{-}+_{-}: C \times C \rightarrow C$ and ${ }_{-} ;_{-}: C \times C \rightarrow C$ (corresponding to the empty process, the prefixing operator, the non-deterministic choice operator and respectively sequential composition).

Proposition 5. For a signature $(\mathrm{C}, \mathrm{F})$, the category $\mathrm{Alg}(\mathrm{T})$ is regular, and the functor $\mathrm{U}_{\mathrm{T}}$ preserves regular-epi-mono factorisations.

The existence of a factorisation system for $\mathrm{Alg}(\mathrm{T})$ yields notions of $\mathrm{T}$-subalgebra, T-homomorphic image and T-variety.

Definition 8. An equation over a signature (C, F) is a T-homomorphic image $q: \mathrm{L}_{\mathrm{T}} X \rightarrow\langle B, \beta\rangle$ with $X$ a RegEpi(C)-projective C-object. A T-algebra $\langle A, \alpha\rangle$ satisfies an equation of this form (written $\langle A, \alpha\rangle=q$ ) if and only if, for any C-arrow $f: X \rightarrow A$, the T-algebra homomorphism $f^{\#}: \mathrm{L}_{\mathrm{T}} X \rightarrow\langle A, \alpha\rangle$ factors through q.

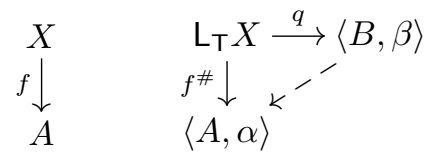

Equations as defined above are an instance of the notion of equation defined in 2. Moreover, equations over a signature are sufficient to characterise varieties. These are consequences of the following observations:

1. If a C-object $X$ is RegEpi(C)-projective (and hence $U_{\mathrm{T}}(\operatorname{RegEpi}(\operatorname{Alg}(\mathrm{T}))$ )projective), then the $\mathrm{T}$-algebra $\mathrm{L}_{\mathrm{T}} X$ is $\operatorname{RegEpi}(\operatorname{Alg}(\mathrm{T}))$-projective.

2. If $C$ has enough RegEpi(C)-projectives, then $\operatorname{Alg}(T)$ has enough $\operatorname{RegEpi}(\operatorname{Alg}(\mathrm{T}))$-projectives. Moreover, the free T-algebras over RegEpi(C)projective C-objects still provide enough $\operatorname{RegEpi(Alg(T)})$-projectives for $\mathrm{Alg}(\mathrm{T})$. 
Remark 4. Given a signature (C, F), equations $q: \mathrm{L}_{\top} X \rightarrow\langle B, \beta\rangle$ over $(\mathrm{C}, \mathrm{F})$ can be specified using pairs of C-arrows $l, r: K \rightarrow \mathrm{U}_{\mathrm{T}} \mathrm{L}_{\mathrm{T}} X$, with $X$ a $\operatorname{RegEpi(C)-~}$ projective $\mathrm{C}$-object. For, such pairs induce pairs of T-algebra homomorphisms $l^{\#}, r^{\#}: \mathrm{L}_{\top} K \rightarrow \mathrm{L}_{\top} X$, whose coequaliser defines an equation over $(\mathrm{C}, \mathrm{F})$. (The preservation of regular epimorphisms by $U_{\mathrm{T}}$ is used to show this.)

$$
\begin{aligned}
& \underset{\mathrm{U}_{\mathrm{T}} \mid \uparrow_{\mathrm{L}_{\mathrm{T}}}}{\mathrm{A}}(\mathrm{T}) \quad \mathrm{L}_{\mathrm{T}} K \underset{r^{\#}}{\stackrel{l^{\#}}{\rightleftarrows}} \mathrm{L}_{\mathrm{T}} X \underset{q}{\stackrel{f^{\#}}{\rightrightarrows}\langle B, \beta\rangle-\rightarrow}\langle A, \alpha\rangle \\
& \stackrel{\mathrm{U}_{\mathrm{T}} \downarrow{ }^{\downarrow} \mathrm{L}_{\mathrm{T}}}{\mathrm{C}} \quad K \underset{r}{\stackrel{l}{\rightleftarrows}} \mathrm{U}_{\mathrm{T}} \mathrm{L}_{\mathrm{T}} X \stackrel{\mathrm{U}_{\mathrm{T}} f^{\#}}{\longrightarrow} A
\end{aligned}
$$

Moreover, a T-algebra $\langle A, \alpha\rangle$ satisfies the induced equation if and only if $\mathrm{U}_{\mathrm{T}} f^{\#}$ 。 $l=\mathrm{U}_{\mathrm{T}} f^{\#} \circ r$ for any $f: X \rightarrow A$. This follows from the definition of $q$ using standard properties of adjunctions.

Equations $q: \mathrm{L}_{\top} X \rightarrow\langle B, \beta\rangle$ with $X$ initial in $\mathrm{C}$ specify properties up to reachability under (ground) computations. Results dual to Props. 2 and 3 hold for algebras of signatures (with the proofs also dualising).

Example 5. The commutativity of the non-deterministic choice operator on processes is formalised by the equation: $(\forall X)(\forall Y) X+Y=Y+X$.

Definition 9. A signature morphism between signatures $(\mathrm{C}, \mathrm{F})$ and $\left(\mathrm{C}^{\prime}, \mathrm{F}^{\prime}\right)$ consists of a pair $(\mathrm{U}, \xi)$ with $\mathrm{U}: \mathrm{C}^{\prime} \rightarrow \mathrm{C}$ a functor with left adjoint $\mathrm{L}$, and with $\xi: \mathrm{F} \circ \mathrm{U} \Rightarrow \mathrm{U} \circ \mathrm{F}^{\prime}$ a natural transformation. $(\mathrm{U}, \xi)$ is horizontal if and only if $\nu_{0^{\prime}} \in \operatorname{RegEpi}(\mathrm{C})$, with $(\mathrm{U}, \nu)$ the induced monad morphism 11 and $0^{\prime}$ initial in $\mathrm{C}^{\prime}$.

Signature morphisms $(U, \xi):(C, F) \rightarrow\left(C^{\prime}, F^{\prime}\right)$ induce reduct functors $U_{\nu}$ : $\operatorname{Alg}\left(\mathrm{T}^{\prime}\right) \rightarrow \operatorname{Alg}(\mathrm{T})$, with $\mathrm{U}_{\nu}$ taking $\left\langle C^{\prime}, \alpha^{\prime}\right\rangle \in\left|\mathrm{Alg}\left(\mathrm{T}^{\prime}\right)\right|$ to $\left\langle\mathrm{U} C^{\prime}, \mathrm{U} \alpha^{\prime} \circ \nu_{C^{\prime}}\right\rangle \in$ $|A| g(T) \mid$. Horizontal signature morphisms capture situations where the target signature does not enrich the notion of reachability induced by the source signature. For, the C-arrow $\nu_{0^{\prime}}: \mathrm{TU} 0^{\prime} \Rightarrow \mathrm{UT}^{\prime} 0^{\prime}$ defines the unique homomorphism from the initial $\mathrm{T}$-algebra to the $\mathrm{U}_{\nu}$-reduct of the initial $\mathrm{T}^{\prime}$-algebra.

Proposition 6. Let $(\mathrm{U}, \xi):(\mathrm{C}, \mathrm{F}) \rightarrow\left(\mathrm{C}^{\prime}, \mathrm{F}^{\prime}\right)$ denote a signature morphism. Then, the reduct functor $\mathrm{U}_{\nu}: \operatorname{Alg}\left(\mathrm{T}^{\prime}\right) \rightarrow \operatorname{Alg}(\mathrm{T})$ has a left adjoint.

Proof. The conclusion follows from $\mathrm{L} \dashv \mathrm{U}, \mathrm{L}_{\mathrm{T}} \dashv \mathrm{U}_{\mathrm{T}}, \mathrm{L}_{\mathrm{T}^{\prime}} \dashv \mathrm{U}_{\mathrm{T}^{\prime}}$ and $\mathrm{U}_{\mathrm{T}} \circ \mathrm{U}_{\nu}=$ $\mathrm{U} \circ \mathrm{U}_{\mathrm{T}^{\prime}}$ using the Adjoint Lifting Theorem (see e.g. [4, Thm. 4.5.6]), after noting that $\operatorname{Alg}\left(\mathrm{T}^{\prime}\right)$ has coequalisers (by Rem.3(1)).

The left adjoint to $\mathrm{U}_{\nu}$ is denoted $\mathrm{L}_{\nu}$. And, as in the coalgebraic case, there exists a natural isomorphism $j: \mathrm{L}_{\mathrm{T}^{\prime}} \circ \mathrm{L} \Rightarrow \mathrm{L}_{\nu} \circ \mathrm{L}_{\mathrm{T}}$.

Signature morphisms also induce translations of equations over their source to equations over their target: an equation $q: \mathrm{L}_{\mathrm{T}} X \rightarrow\langle B, \beta\rangle$ translates along a signature morphism $(\mathrm{U}, \xi):(\mathrm{C}, \mathrm{F}) \rightarrow\left(\mathrm{C}^{\prime}, \mathrm{F}^{\prime}\right)$ to the equation $\nu(q)=\mathrm{L}_{\nu} q \circ j_{X}$.

${ }^{11}$ The natural transformation $\nu: \mathrm{T} \circ \mathrm{U} \Rightarrow \mathrm{U} \circ \mathrm{T}^{\prime}$ is obtained similarly to the natural transformation $\rho: \mathrm{U} \circ \mathrm{D}^{\prime} \Rightarrow \mathrm{D} \circ \mathrm{U}$ in Rem.2. 
Remark 5. A signature morphism $(\mathrm{U}, \xi):(\mathrm{C}, \mathrm{F}) \rightarrow\left(\mathrm{C}^{\prime}, \mathrm{F}^{\prime}\right)$ also induces a mapping from pairs of $\mathrm{C}$-arrows defining equations over (C,F) (see Rem.4) to pairs of $\mathrm{C}^{\prime}$-arrows defining equations over $\left(\mathrm{C}^{\prime}, \mathrm{F}^{\prime}\right)$. The mapping takes a pair $l, r: K \rightarrow$ $\mathrm{U}_{\mathrm{T}} \mathrm{L}_{\mathrm{T}} X$ to the pair $\left(\mathrm{UU}_{\mathrm{T}^{\prime}} j_{X}^{-1} \circ \mathrm{U}_{\mathrm{T}} \eta_{\nu, \mathrm{L}_{\mathrm{T}} X} \circ l\right)^{\#},\left(\mathrm{UU}_{\mathrm{T}^{\prime}} j_{X}^{-1} \circ \mathrm{U}_{\mathrm{T}} \eta_{\nu, \mathrm{L}_{\mathrm{T}} X} \circ r\right)^{\#}: \mathrm{L} K \rightarrow$ $\mathrm{U}_{\mathrm{T}^{\prime}} \mathrm{L}_{\mathrm{T}^{\prime}} \mathrm{L} X$, where $\eta_{\nu}: \mathrm{Id} \Rightarrow \mathrm{U}_{\nu} \circ \mathrm{L}_{\nu}$ denotes the unit of the adjunction $\mathrm{L}_{\nu} \dashv \mathrm{U}_{\nu}$.

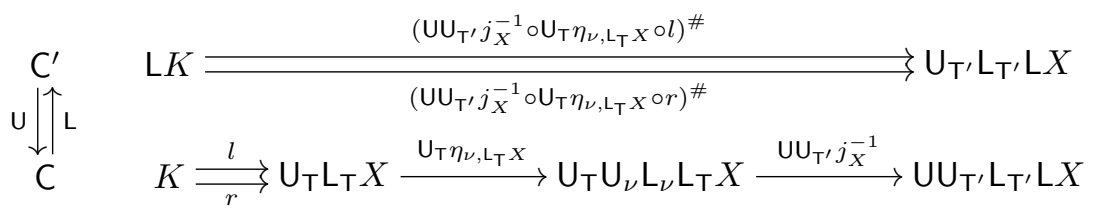

Moreover, the mapping thus defined agrees with the translation of equations over $(C, F)$ to equations over $\left(C^{\prime}, F^{\prime}\right)$. That is, the translation along $(U, \xi)$ of the equation induced by $l, r$ coincides (up to isomorphism in $\operatorname{Alg}\left(T^{\prime}\right)$ ) with the equation induced by $\left(\mathrm{UU}_{\mathrm{T}^{\prime}} j_{X}^{-1} \circ \mathrm{U}_{\mathrm{T}} \eta_{\nu, \mathrm{L}_{\mathrm{T}} X} \circ l\right)^{\#},\left(\mathrm{UU}_{\mathrm{T}^{\prime}} j_{X}^{-1} \circ \mathrm{U}_{\mathrm{T}} \eta_{\nu, \mathrm{L}_{\mathrm{T}} X} \circ r\right)^{\#}$. This follows using standard properties of adjunctions.

Theorem 2. Let $(\mathrm{U}, \xi):(\mathrm{C}, \mathrm{F}) \rightarrow\left(\mathrm{C}^{\prime}, \mathrm{F}^{\prime}\right)$ denote a signature morphism, let $\left\langle A^{\prime}, \alpha^{\prime}\right\rangle$ denote a $\mathrm{T}^{\prime}$-algebra, and let $q: \mathrm{L}_{\top} X \rightarrow\langle B, \beta\rangle$ denote an equation over $(\mathrm{C}, \mathrm{F})$. Then, $\mathrm{U}_{\nu}\left\langle A^{\prime}, \alpha^{\prime}\right\rangle \models q$ iff $\left\langle A^{\prime}, \alpha^{\prime}\right\rangle \models \nu(q)$.

\section{Combined Structures and Their Logic}

The frameworks described in Sections 2 and 3 are now integrated in order to account for structures incorporating both algebraic and coalgebraic features. Modal as well as equational formulae are used to formalise correctness properties of such structures, with the associated notions of satisfaction abstracting away unreachable and respectively observationally indistinguishable behaviours. Such an abstraction is possible due to a compatibility between computational and observational features in the structures considered. This compatibility, which amounts to computations preserving observational indistinguishability and to observations preserving reachability, is attained using an approach similar to that of [17], where liftings of monads to categories of coalgebras are used to define operational semantics which are well-behaved w.r.t. denotational ones.

Definition 10. A combined signature is a tuple (C, G, F, $\sigma$ ), with (C, G) a cosignature, $(\mathrm{C}, \mathrm{F})$ a signature inducing a monad $(\mathrm{T}, \eta, \mu)$, and $\sigma: \mathrm{T} \circ \mathrm{U}_{\mathrm{G}} \Rightarrow$ $\mathrm{G} \circ \mathrm{T} \circ \mathrm{U}_{\mathrm{G}}$ a natural transformation, such that the following diagram commutes:

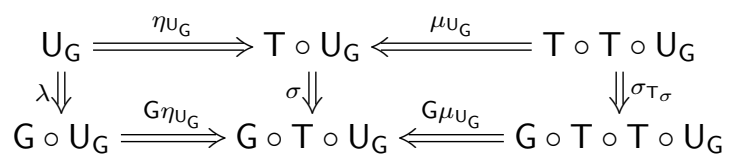

where the natural transformation $\lambda: \mathrm{U}_{\mathrm{G}} \Rightarrow \mathrm{G} \circ \mathrm{U}_{\mathrm{G}}$ is given by $\lambda_{\gamma}=\gamma$ for $\langle C, \gamma\rangle \in|\operatorname{Coalg}(\mathrm{G})|$, while the functor $\mathrm{T}_{\sigma}: \operatorname{Coalg}(\mathrm{G}) \rightarrow \operatorname{Coalg}(\mathrm{G})$ is given by $\mathrm{T}_{\sigma}\langle C, \gamma\rangle=\left\langle\mathrm{T} C, \sigma_{\gamma}\right\rangle$ for $\langle C, \gamma\rangle \in|\mathrm{Coalg}(\mathrm{G})|$ (and consequently $\mathrm{U}_{\mathrm{G}} \circ \mathrm{T}_{\sigma}=\mathrm{T}_{0} \mathrm{U}_{\mathrm{G}}$ ). 
A combined signature morphism from $(\mathrm{C}, \mathrm{G}, \mathrm{F}, \sigma)$ to $\left(\mathrm{C}^{\prime}, \mathrm{G}^{\prime}, \mathrm{F}^{\prime}, \sigma^{\prime}\right)$ is a tuple $(\mathrm{U}, \tau, \xi)$, with $(\mathrm{U}, \tau):(\mathrm{C}, \mathrm{G}) \rightarrow\left(\mathrm{C}^{\prime}, \mathrm{G}^{\prime}\right)$ a cosignature morphism and $(\mathrm{U}, \xi)$ : $(\mathrm{C}, \mathrm{F}) \rightarrow\left(\mathrm{C}^{\prime}, \mathrm{F}^{\prime}\right)$ a signature morphism inducing a monad morphism $(\mathrm{U}, \nu)$ : $(\mathrm{T}, \eta, \mu) \rightarrow\left(\mathrm{T}^{\prime}, \eta^{\prime}, \mu^{\prime}\right)$, such that the following diagram commutes:

$$
\begin{aligned}
& \mathrm{T} \circ \mathrm{U} \circ \mathrm{U}_{\mathrm{G}^{\prime}}=\mathrm{T} \circ \mathrm{U}_{\mathrm{G}} \circ \mathrm{U}_{\tau} \Longrightarrow \mathrm{G} \circ \mathrm{\textrm {U } _ { \tau }} / \mathrm{U}_{\mathrm{G}} \circ \mathrm{U}_{\tau}=\mathrm{G} \circ \mathrm{T} \circ \mathrm{U} \circ \mathrm{U}_{\mathrm{G}^{\prime}} \\
& \mathrm{U} \circ \mathrm{T}^{\prime} \circ \mathrm{U}_{\mathrm{G}^{\prime}} \stackrel{\mathrm{U} \sigma^{\prime}}{\Longrightarrow} \mathrm{U} \circ \mathrm{G}^{\prime} \circ \mathrm{T}^{\prime} \circ \mathrm{U}_{\mathrm{G}^{\prime}} \stackrel{\tau_{\mathrm{T}^{\prime} \circ \mathrm{U}_{\mathrm{G}^{\prime}}}}{\Longrightarrow} \mathrm{G} \circ \mathrm{U} \circ \mathrm{T}^{\prime} \circ \mathrm{U}_{\mathrm{G}^{\prime}}
\end{aligned}
$$

The natural transformation $\sigma$ used in the definition of combined signatures specifies the relationship between the algebraic and coalgebraic substructures of combined structures. Its components define G-coalgebra structures on (the carriers of) the free T-algebras over (the carriers of) G-coalgebras 4 . The additional constraints on $\sigma$ ensure that, for any $\mathrm{G}$-coalgebra $\langle C, \gamma\rangle$, the $\mathrm{C}$-arrows $\eta_{C}: C \rightarrow \mathrm{T} C$ and $\mu_{C}: \mathrm{TT} C \rightarrow C$ define G-coalgebra homomorphisms. This results in the tuple $\left(\mathrm{T}_{\sigma}, \eta, \mu\right)$ defining a monad on Coalg $(\mathrm{G})$. The algebras of this monad are taken as models for a combined signature (C, G, F, $\sigma)$. A $\mathrm{T}_{\sigma}$-algebra is thus given by a C-object $C$ carrying both a $\mathrm{G}$-coalgebra structure $\langle C, \gamma\rangle$ and a T-algebra structure $\langle C, \alpha\rangle$, such that $\alpha$ defines a G-coalgebra homomorphism from $\left\langle\mathrm{T} C, \sigma_{\gamma}\right\rangle$ to $\langle C, \gamma\rangle$. Then, the constraints defining a combined signature morphism $(\mathrm{U}, \tau, \xi)$ ensure that, for a $\mathrm{G}^{\prime}$-coalgebra $\left\langle C^{\prime}, \gamma^{\prime}\right\rangle$, the $\mathrm{G}^{\prime}$-coalgebra structure induced by $\sigma^{\prime}$ on $\mathrm{T}^{\prime} C^{\prime}$ agrees with the G-coalgebra structure induced by $\sigma$ on TU $C^{\prime}$. This results in combined signature morphisms $(\mathrm{U}, \tau, \xi)$ inducing reduct functors $\mathrm{U}_{(\tau, \nu)}: \operatorname{Alg}\left(\mathrm{T}_{\sigma^{\prime}}^{\prime}\right) \rightarrow \operatorname{Alg}\left(\mathrm{T}_{\sigma}\right)$, with $\mathrm{U}_{(\tau, \nu)}$ taking a $\mathrm{T}_{\sigma^{\prime}}^{\prime}$-algebra $\left\langle\left\langle C^{\prime}, \gamma^{\prime}\right\rangle, \alpha^{\prime}\right\rangle$ to the $\mathrm{T}_{\sigma}$-algebra $\left\langle\left\langle\mathrm{U} C^{\prime}, \tau_{C^{\prime}} \circ \mathrm{U} \gamma^{\prime}\right\rangle, \mathrm{U} \alpha^{\prime} \circ \nu_{C^{\prime}}\right\rangle$.

Remark 6. In 1211, combined structures are captured using pairs consisting of an algebra and a coalgebra structure on the same carrier, with the additional requirement that bisimulation on the coalgebraic structure is a congruence w.r.t. the algebraic structure. Here, the presence of natural transformations $\sigma$ in the definition of combined signatures ensures this, as well as the fact that reachable subalgebras carry coalgebraic structure. Another consequence of the use of such natural transformations is the existence of reduct functors induced by combined signature morphisms. An alternative approach to specifying combined structures would be to require the above-mentioned compatibility between the algebraic and coalgebraic structure directly, rather than ensuring it through the natural transformations $\sigma$. Formally, this would amount to requiring the reachable subalgebras of the underlying algebras to carry coalgebraic structure, and the observable homomorphic images of the underlying coalgebras to carry algebraic structure 13 . However, in this case, horizontality of the signature and cosignature morphisms used to define combined signature morphisms would be

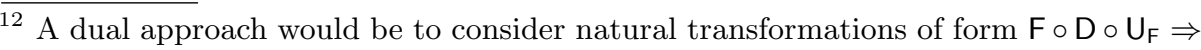
$\mathrm{D} \circ \mathrm{U}_{\mathrm{F}}$, with $\mathrm{D}$ the comonad induced by $\mathrm{G}$.

13 The existence of factorisation systems for $\operatorname{Alg}(T)$ and Coalg $(G)$ results in the reachable subalgebra of a T-algebra and the observable homomorphic image of a G-coalgebra being defined uniquely up to isomorphism in $\operatorname{Alg}(T)$ and respectively Coalg $(G)$. 
needed to ensure the well-definedness of reduct functors. (11 also uses a condition which resembles horizontality to ensure that reduct functors preserve the compatibility between the algebraic operations and the bisimulation relation.)

Example 6. Non-deterministic, sequential processes are specified using the combined signature (Set, $\mathrm{G}_{\text {LFTS }}, \mathrm{F}_{\mathrm{NSP}}, \sigma$ ), with $\sigma: \mathrm{T}_{\text {NSP }} \circ \mathrm{U} \Rightarrow \mathrm{G}_{\text {LFTS }} \circ \mathrm{T}_{\mathrm{NSP}} \circ \mathrm{U}$ (where $\mathrm{T}_{\mathrm{NSP}}$ denotes the monad induced by $\mathrm{F}_{\mathrm{NSP}}$ ) being defined inductively by:

$$
\begin{aligned}
\sigma_{\gamma}(c) & =\langle\operatorname{next}(c), \operatorname{depth}(c)\rangle \\
\sigma_{\gamma}(\operatorname{nil}) & =\langle\emptyset, 0\rangle \\
\sigma_{\gamma}(a . c) & =\langle\{\langle a, c\rangle\}, 1+\operatorname{depth}(c)\rangle \\
\sigma_{\gamma}(c+d) & =\langle\operatorname{next}(c) \cup \operatorname{next}(d), \max (\operatorname{depth}(c), \operatorname{depth}(d))\rangle \\
\sigma_{\gamma}(c ; d) & =\left\{\begin{array}{l}
\langle\operatorname{next}(d), \operatorname{depth}(d)\rangle, \operatorname{if} \operatorname{depth}(c)=0 \\
\left\langle\left\{\left\langle a, c^{\prime} ; d\right\rangle \mid\left\langle a, c^{\prime}\right\rangle \in \operatorname{next}(c)\right\}, \operatorname{depth}(c)+\operatorname{depth}(d)\right\rangle, \mathrm{o} / \mathrm{w}
\end{array}\right.
\end{aligned}
$$

for any $\mathrm{G}_{\mathrm{LFTS}}$-coalgebra $\langle C,\langle$ next, depth $\rangle\rangle, a \in A$ and $c, d \in C$.

It follows from 17] that, for a combined signature $(C, G, F, \sigma)$, the category $\operatorname{Alg}\left(\mathrm{T}_{\sigma}\right)$ has both an initial and a final object. The initial $\mathrm{T}_{\sigma}$-algebra provides an observational structure on ground computations, whereas the final $\mathrm{T}_{\sigma}$-algebra provides a computational structure on abstract states. These $\mathrm{T}_{\sigma}$-algebras will, from now on, be denoted $\left\langle\left\langle I, \gamma_{I}\right\rangle, \alpha_{I}\right\rangle$ and respectively $\left\langle\left\langle F, \gamma_{F}\right\rangle, \alpha_{F}\right\rangle$.

Proposition 7. For a combined signature (C, G, F, $\sigma$ ), the factorisation system for Coalg $(\mathrm{G})$ given by Prop. 1 lifts uniquely to a factorisation system for $\operatorname{Alg}\left(\mathrm{T}_{\sigma}\right)$. Moreover, the functor taking $\mathrm{T}_{\sigma}$-algebras to their underlying $\mathrm{T}$-algebras preserves factorisations.

Proof. The first statement follows by [1, Prop. 20.28], after noting that $\mathrm{T}_{\sigma}$ preserves regular epimorphisms (as $\mathrm{T}$ preserves regular epimorphisms). For the second statement, let $\mathrm{U}_{\sigma}^{\mathrm{G}}: \operatorname{Alg}\left(\mathrm{T}_{\sigma}\right) \rightarrow \operatorname{Coalg}(\mathrm{G})$ and $\mathrm{U}_{\sigma}^{\mathrm{T}}: \operatorname{Alg}\left(\mathrm{T}_{\sigma}\right) \rightarrow \operatorname{Alg}(\mathrm{T})$ denote the functors taking $\mathrm{T}_{\sigma}$-algebras to their underlying $\mathrm{G}$-coalgebras and respectively $\mathrm{T}$-algebras. Then, the statement follows from $\mathrm{U}_{\mathrm{G}} \circ \mathrm{U}_{\sigma}^{\mathrm{G}}=\mathrm{U}_{\mathrm{T}} \circ \mathrm{U}_{\sigma}^{\mathrm{T}}$, together with $U_{G} \circ U_{\sigma}^{G}$ preserving factorisations and $U_{T}$ creating them.

As a consequence of Prop.7, the observable homomorphic images of the Gcoalgebras underlying $\mathrm{T}_{\sigma}$-algebras carry $\mathrm{T}$-algebra structure, whereas the reachable subalgebras of the $\mathrm{T}$-algebras underlying $\mathrm{T}_{\sigma}$-algebras carry $\mathrm{G}$-coalgebra structure.

Definition 11. Let $(\mathrm{C}, \mathrm{G}, \mathrm{F}, \sigma)$ denote a combined signature. A $\mathrm{T}_{\sigma}$-algebra $\langle\langle C, \gamma\rangle, \alpha\rangle$ satisfies a modal formula $\iota:\langle D, \delta\rangle \rightarrow \mathrm{R}_{\mathrm{G}} Z$ over $(\mathrm{C}, \mathrm{G})$ up to reachability (written $\langle\langle C, \gamma\rangle, \alpha\rangle \models^{\mathrm{r}} \iota$ ) if and only if, for any $\mathrm{C}$-arrow $f: C \rightarrow Z$, the $\mathrm{G}$-coalgebra homomorphism $f^{b} \circ !:\left\langle I, \gamma_{I}\right\rangle \rightarrow \mathrm{R}_{\mathrm{G}} Z$, with $!:\left\langle I, \gamma_{I}\right\rangle \rightarrow\langle C, \gamma\rangle$ arising from the initiality of $\left\langle\left\langle I, \gamma_{I}\right\rangle, \alpha_{I}\right\rangle$, factors through $\iota$.

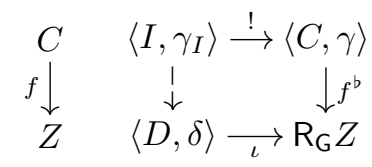


Also, a $\mathrm{T}_{\sigma}$-algebra $\langle\langle C, \gamma\rangle, \alpha\rangle$ satisfies an equation $q: \mathrm{L}_{\top} X \rightarrow\langle B, \beta\rangle$ over $(\mathrm{C}, \mathrm{F})$ up to observability (written $\langle\langle C, \gamma\rangle, \alpha\rangle \models^{\circ} q$ ) if and only if, for any C-arrow $f: X \rightarrow C$, the T-algebra homomorphism !'。 $f^{\#}: \mathrm{L}_{\mathrm{T}} X \rightarrow\left\langle F, \alpha_{F}\right\rangle$, with $!^{\prime}:\langle C, \alpha\rangle \rightarrow\left\langle F, \alpha_{F}\right\rangle$ arising from the finality of $\left\langle\left\langle F, \gamma_{F}\right\rangle, \alpha_{F}\right\rangle$, factors through $q$.

$$
\begin{array}{clc}
X & \mathrm{~L}_{\mathrm{T}} X \stackrel{q}{\longrightarrow}\langle B, \beta\rangle \\
f \downarrow & f^{\#} \downarrow & \downarrow \\
\stackrel{\downarrow}{C} & \langle C, \alpha\rangle \underset{! !}{\longrightarrow}\left\langle F, \alpha_{F}\right\rangle
\end{array}
$$

Remark 7. If the equation $q$ in Def.11] is given by a pair of C-arrows $l, r: K \rightarrow$ $\mathrm{U}_{\mathrm{T}} \mathrm{L}_{\mathrm{T}} X$, then $\langle\langle C, \gamma\rangle, \alpha\rangle={ }^{\circ} q$ translates to $\mathrm{U}_{\mathrm{T}} !^{\prime} \circ \mathrm{U}_{\mathrm{T}} f^{\#} \circ l=\mathrm{U}_{\mathrm{T}} !^{\prime} \circ \mathrm{U}_{\mathrm{T}} f^{\#} \circ r$ for any C-arrow $f: X \rightarrow C$. This follows similarly to Rem.4.

Remark 8. The notions of satisfaction introduced in Def.11 can also be defined in a more general setting, which does not assume the existence of initial/final $\mathrm{T}_{\sigma}$-algebras. In particular, in a setting where combined structures are given by compatible algebra-coalgebra pairs (see Rem.6), a notion of satisfaction of modal formulae up to reachability is obtained by replacing the G-coalgebra homomorphism ! : $\left\langle I, \gamma_{I}\right\rangle \rightarrow\langle C, \gamma\rangle$ in Def.11] with the G-coalgebra homomorphism $r:\left\langle R, \gamma_{R}\right\rangle \rightarrow\langle C, \gamma\rangle$ (with $\left\langle R, \gamma_{R}\right\rangle$ denoting the G-coalgebra structure on the carrier of the reachable T-subalgebra of $\langle C, \alpha\rangle \sqrt{14})$. A notion of satisfaction of equations up to observability can be defined in a similar way.

The satisfaction of a modal formula by a $\mathrm{T}_{\sigma}$-algebra only requires the formula to hold in reachable states. Also, as shown by the next result, the satisfaction of an equation by a $T_{\sigma}$-algebra only requires the equation to hold up to bisimilarity.

Proposition 8. Let (C, G, F, $\sigma$ ) denote a combined signature. Then, a $\mathrm{T}_{\sigma}$-algebra $\langle\langle C, \gamma\rangle, \alpha\rangle$ satisfies an equation $l, r: K \rightarrow \mathrm{U}_{\mathrm{T}} \mathrm{L}_{\mathrm{T}} X$ over $(\mathrm{C}, \mathrm{F})$ up to observability if and only if, for any $\mathrm{C}$-arrow $f: X \rightarrow C,\left\langle\mathrm{U}_{\mathrm{T}} f^{\#} \circ l, \mathrm{U}_{\mathrm{T}} f^{\#} \circ r\right\rangle$ factors through $\left\langle\pi_{1}, \pi_{2}\right\rangle$, with $\pi_{1}, \pi_{2}: R \rightarrow C$ defining the kernel pair of $\mathrm{U}_{\mathrm{T}} ! 15$.

Proof (sketch). Rem.7] is used.

The next result gives a necessary and sufficient condition for the satisfaction of modal formulae up to reachability by algebras of combined signatures.

Proposition 9. Let (C, G, F, $\sigma)$ denote a combined signature, let $\langle\langle C, \gamma\rangle, \alpha\rangle$ denote a $\mathrm{T}_{\sigma}$-algebra with $\left\langle\left\langle R, \gamma_{R}\right\rangle, \alpha_{R}\right\rangle$ its reachable $\mathrm{T}_{\sigma}$-subalgebra, and let $\iota$ : $\langle D, \delta\rangle \rightarrow \mathrm{R}_{\mathrm{G}} Z$ denote a modal formula over $(\mathrm{C}, \mathrm{G})$. Then, $\langle\langle C, \gamma\rangle, \alpha\rangle \models^{\mathrm{r}} \iota$ iff $\left\langle R, \gamma_{R}\right\rangle \models \iota$.

Proof (sketch). The unique (RegEpi(Coalg $(\mathrm{G}))$, Mono(Coalg $(\mathrm{G}))$ )-diagonalisation property of Coalg $(\mathrm{G})$ and the injectivity of $Z$ are used for the only if direction.

${ }^{14}$ The preservation of monomorphisms by $\mathrm{G}$ ensures the uniqueness of $\left\langle R, \gamma_{R}\right\rangle$.

${ }^{15}$ Hence, by Rem.1(4),$\left\langle R, \pi_{1}, \pi_{2}\right\rangle$ gives precisely the bisimilarity relation on $\langle C, \gamma\rangle$. 
A similar result holds for the satisfaction of equations up to observability: a $\mathrm{T}_{\sigma}$-algebra satisfies an equation up to observability if and only if the algebra underlying its observable homomorphic image satisfies that equation.

Initiality yields an inductive technique for proving the satisfaction of modal formulae up to reachability by algebras of combined signatures. Also, finality together with the existence of largest bisimulations yield a coinductive technique for proving the satisfaction of equations up to observability by algebras of combined signatures. These techniques are briefly illustrated in the following.

Example \%. Proving that the modal formulae defining finitely-branching, $A$ labelled transition systems of finite depth hold, up to reachability, in algebras of the combined signature for non-deterministic, sequential processes can be reduced to proving that they hold in nil and that their satisfaction is preserved

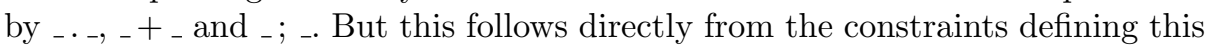
combined signature. Also, proving that the equation in Ex.5 holds, up to observability, in algebras of the same combined signature can be reduced to exhibiting a generic bisimulation relation (given by a functor $\mathrm{R}: \operatorname{Coalg}\left(\mathrm{G}_{\mathrm{LFTS}}\right) \rightarrow \mathrm{C}$ together with two natural transformations $\pi_{1}, \pi_{2}: \mathrm{R} \Rightarrow \mathrm{U}_{\mathrm{G}}$, such that $\left\langle\mathrm{R} \gamma, \pi_{1, \gamma}, \pi_{2, \gamma}\right\rangle$ is a bisimulation relation on $\langle C, \gamma\rangle$ for each $\mathrm{G}_{\mathrm{LFTS}}$-coalgebra $\langle C, \gamma\rangle$ ), such that $\mathrm{R} \gamma$ relates the interpretations in $\langle C, \alpha\rangle$ of the two sides of the equation, for any $\left(\mathrm{G}_{\mathrm{LFTS}}, \mathrm{F}_{\mathrm{NSP}}, \sigma\right)$-algebra $\langle\langle C, \gamma\rangle, \alpha\rangle$. Here, $\mathrm{R} \gamma$ is taken to be the least reflexive relation on $C$ such that $(c+d) \mathrm{R} \gamma(d+c)$ for any $c, d \in C$ and any $\left(\mathrm{G}_{\mathrm{LFTS}}, \mathrm{F}_{\mathrm{NSP}}, \sigma\right)$ algebra $\langle\langle C, \gamma\rangle, \alpha\rangle$. The fourth constraint in the definition of this combined signature results in $\mathrm{R} \gamma$ being a bisimulation relation on $\langle C, \gamma\rangle$.

Finally, we show that combined signature morphisms whose underlying signature and respectively cosignature morphisms are horizontal give rise to institutions w.r.t. the satisfaction of modal formulas up to reachability and respectively of equations up to observability by algebras of combined signatures.

Theorem 3. Let $(\mathrm{U}, \tau, \xi):(\mathrm{C}, \mathrm{G}, \mathrm{F}, \sigma) \rightarrow\left(\mathrm{C}^{\prime}, \mathrm{G}^{\prime}, \mathrm{F}^{\prime}, \sigma^{\prime}\right)$ denote a combined signature morphism, and let $\left\langle\left\langle C^{\prime}, \gamma^{\prime}\right\rangle, \alpha^{\prime}\right\rangle$ denote a $\mathrm{T}_{\sigma^{\prime}}^{\prime}$-algebra. The following hold:

1. If $(\mathrm{U}, \xi)$ is horizontal and $\iota:\langle D, \delta\rangle \rightarrow \mathrm{R}_{\mathrm{G}} Z$ denotes a modal formula over $(\mathrm{C}, \mathrm{G})$, then $\mathrm{U}_{\tau, \nu}\left\langle\left\langle C^{\prime}, \gamma^{\prime}\right\rangle, \alpha^{\prime}\right\rangle \models^{\mathrm{r}} \iota$ iff $\left\langle\left\langle C^{\prime}, \gamma^{\prime}\right\rangle, \alpha^{\prime}\right\rangle \models{ }^{\mathrm{r}} \tau(\iota)$.

2. If $(\mathrm{U}, \tau)$ is horizontal and $q: \mathrm{L}_{\top} X \rightarrow\langle B, \beta\rangle$ denotes an equation over $(\mathrm{C}, \mathrm{F})$, then $\mathrm{U}_{\tau, \nu}\left\langle\left\langle C^{\prime}, \gamma^{\prime}\right\rangle, \alpha^{\prime}\right\rangle \models{ }^{\circ} q$ iff $\left\langle\left\langle C^{\prime}, \gamma^{\prime}\right\rangle, \alpha^{\prime}\right\rangle \models{ }^{\circ} \nu(q)$.

Proof. $\mathrm{U}_{\tau, \nu}\left\langle\left\langle C^{\prime}, \gamma^{\prime}\right\rangle, \alpha^{\prime}\right\rangle=^{\mathrm{r}} \iota$ amounts to the existence, for any C-arrow $f$ : $\mathrm{U} C^{\prime} \rightarrow Z$, of a G-coalgebra homomorphism $g$ making the outer left diagram below commute, whereas $\left\langle\left\langle C^{\prime}, \gamma^{\prime}\right\rangle, \alpha^{\prime}\right\rangle \models^{r} \tau(\iota)$ amounts to the existence, for any $\mathrm{C}^{\prime}$-arrow $f^{\prime}: C^{\prime} \rightarrow \mathrm{R} Z$, of a $\mathrm{G}^{\prime}$-coalgebra homomorphism $g^{\prime}$ making the right diagram below commute. Also, since $\mathrm{U} \dashv \mathrm{R}, \mathrm{C}\left(\mathrm{U} C^{\prime}, Z\right) \simeq \mathrm{C}^{\prime}\left(C^{\prime}, \mathrm{R} Z\right)$.

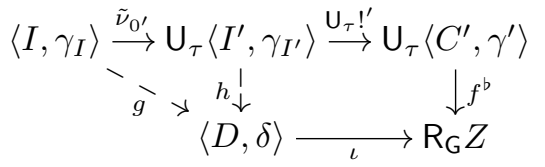

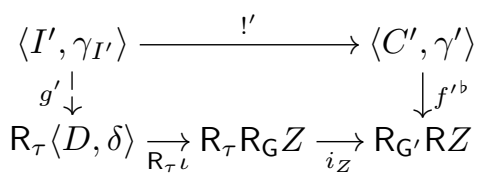


For the if direction, let $f: \mathrm{U} C^{\prime} \rightarrow Z$ denote a C-arrow, let $f^{\prime}: C^{\prime} \rightarrow \mathrm{R} Z$ denote its corresponding $C^{\prime}$-arrow, and let $g^{\prime}:\left\langle I^{\prime}, \gamma_{I^{\prime}}\right\rangle \rightarrow \mathrm{R}_{\tau}\langle D, \delta\rangle$ denote the $\mathrm{G}^{\prime}$-coalgebra homomorphism which makes the right diagram commute. Then, $g$ is taken to be $g^{\prime \#} \circ \tilde{\nu}_{0^{\prime}}$. For the only if direction, let $f^{\prime}: C^{\prime} \rightarrow \mathrm{R} Z$ denote a $\mathrm{C}^{\prime}$-arrow, and let $f: \mathrm{U}^{\prime} \rightarrow Z$ denote its corresponding $\mathrm{C}$-arrow. Since $\mathrm{U}_{\mathrm{G}} \tilde{\nu}_{0^{\prime}}=\nu_{0^{\prime}} \in \operatorname{RegEpi}(\mathrm{C})$ and $\mathrm{U}_{\mathrm{G}} \iota \in \operatorname{Mono}(\mathrm{C})$, the unique (RegEpi $(\operatorname{Coalg}(\mathrm{G})$ ), Mono(Coalg $(\mathrm{G}))$ )-diagonalisation property of Coalg $(\mathrm{G})$ yields a $\mathrm{G}$-coalgebra homomorphism $h: \mathrm{U}_{\tau}\left\langle I^{\prime}, \gamma_{I^{\prime}}\right\rangle \rightarrow\langle D, \delta\rangle$ satisfying $h \circ \tilde{\nu}_{0^{\prime}}=g$ and $\iota \circ h=f^{b} \circ \mathrm{U}_{\tau} !^{\prime}$. This, in turn, yields a $\mathrm{G}^{\prime}$-coalgebra homomorphism $g^{\prime}:\left\langle I^{\prime}, \gamma_{I^{\prime}}\right\rangle \rightarrow \mathrm{R}_{\tau}\langle D, \delta\rangle$ (by $\left.\mathrm{U}_{\tau} \dashv \mathrm{R}_{\tau}\right)$.

The proof of the second statement is similar.

\section{References}

1. J. Adámek, H. Herrlich, and G.E. Strecker. Abstract and Concrete Categories. John Wiley and Sons, 1990.

2. B. Banaschewski and H. Herrlich. Subcategories defined by implications. Houston Journal of Mathematics, 2(2), 1976.

3. G. Birkhoff. On the structure of abstract algebras. In Proceedings of the Cambridge Philosophical Society, volume 31, 1935.

4. F. Borceux. Handbook of Categorical Algebra, volume 2. CUP, 1994.

5. C. Cîrstea. Integrating observational and computational features in the specification of state-based, dynamical systems. Theoretical Informatics and Applications, 35(1), 2001.

6. J. Goguen and R. Burstall. Institutions: Abstract model theory for specification and programming. Journal of the ACM, 39(1), 1992.

7. B. Jacobs. Many-sorted coalgebraic modal logic: A model-theoretic study. Theoretical Informatics and Applications, 35(1), 2001.

8. P. Johnstone, J. Power, T. Tsujishita, H. Watanabe, and J. Worrell. On the structure of categories of coalgebras. TCS, 260(1), 2001.

9. A. Kurz. Logics for Coalgebras and Applications to Computer Science. PhD thesis, Ludwig-Maximilians-Universität München, 2000.

10. A. Kurz. Specifying coalgebras with modal logic. TCS, 260(1), 2001.

11. A. Kurz and R. Hennicker. On institutions for modular coalgebraic specifications. To appear in Theoretical Computer Science.

12. G. Malcolm. Behavioural equivalence, bisimulation, and minimal realisation. In M. Haveraaen, O. Owe, and O.-J. Dahl, editors, Proceedings, WADT, volume 1130 of LNCS. Springer, 1996.

13. L.S. Moss. Coalgebraic logic. Annals of Pure and Applied Logic, 96, 1999.

14. H. Reichel. An approach to object semantics based on terminal coalgebras. Mathematical Structures in Computer Science, 5, 1995.

15. M. Rößiger. Coalgebras and modal logic. In H. Reichel, editor, Coalgebraic Methods in Computer Science, volume 33 of ENTCS. Elsevier Science, 2000.

16. J.J.M.M. Rutten. Universal coalgebra: a theory of systems. TCS, 249(1), 2000.

17. D. Turi and G. Plotkin. Towards a mathematical operational semantics. In Proceedings, LICS, 1997. 